\title{
Expression of vascular endothelial growth factor and caspase-3 in mucinous breast carcinoma and infiltrating ductal carcinoma-not otherwise specified, and the correlation with disease-free survival
}

\author{
QIULI WANG ${ }^{1}$, LISHA SUN ${ }^{1}$, JICI YAN $^{1}$, SHUO WANG $^{1}$, JUNCHENG ZHANG $^{1}$ and XINYU ZHENG ${ }^{1,2}$ \\ ${ }^{1}$ Department of Breast Surgery, First Affiliated Hospital; ${ }^{2}$ Laboratory 1, Cancer Institute, First Affiliated Hospital, \\ China Medical University, Shenyang, Liaoning 110001, P.R. China
}

Received January 22, 2016; Accepted June 2, 2017

DOI: $10.3892 / \mathrm{ol} .2017 .6744$

\begin{abstract}
Mucinous breast carcinoma (MBC) is a rare type of breast cancer, but it has been infrequently studied due to its associated good prognosis. Vascular endothelial growth factor (VEGF) and caspase-3 have been identified to be prognostic factors of infiltrating ductal carcinoma-not otherwise specified (IDC-NOS), but their expression in MBC has not been reported. In the present study, the expression of caspase-3 and VEGF in MBC and IDC-NOS were assessed by immunohistochemistry. Scoring was conducted based on staining intensity and percentage of positive cells. Based on the scores of caspase-3 and VEGF expression, all patient samples were divided into two groups: Low expression (score of 0-5) or high expression (score of 6-12). In total, $42.59 \%$ of MBC patients exhibited a high VEGF score compared with $61.67 \%$ of the IDC-NOS group $(\mathrm{P}<0.05)$. Furthermore, $57.41 \%$ of MBC patients exhibited high caspase- 3 expression compared with only $33.33 \%$ of IDC-NOS patients $(\mathrm{P}<0.05)$. VEGF expression in MBC was associated with age, nodal status and tumor-node-metastasis (TNM) stage. Cox univariate analysis showed that higher VEGF expression, positive nodal status and higher TNM stage were associated with shorter disease-free survival (DFS). The Kaplan-Meier method showed that higher VEGF expression in MBC was associated with worse DFS times, while Cox multivariate analysis showed that only TNM stage was significantly associated with DFS. VEGF and caspase-3 expression varied in the MBC and IDC-NOS samples, but neither was directly correlated with DFS in the MBC patients.
\end{abstract}

Correspondence to: Dr Xinyu Zheng, Department of Breast Surgery, First Affiliated Hospital, China Medical University, 155 North Nanjing Street, Shenyang, Liaoning 110001, P.R. China E-mail: xyzheng@cmu.edu.cn

Key words: mucinous breast carcinoma, infiltrating ductal carcinoma-not otherwise specified, vascular endothelial growth factor, caspase-3, disease-free survival

\section{Introduction}

Mucinous breast carcinoma (MBC), also known as colloid carcinoma, is a rare subtype of breast tumors that accounts for $1-7 \%$ of all breast cancer cases. $\mathrm{MBC}$ is characterized by the presence of extracellular mucin (MUC) (1). MBC includes mixed $\mathrm{MBC}$, consisting of other cancer types such as invasive ductal carcinoma, and pure MBC (PMBC), in which the entire mass is almost occupied by mucinous cancer cells and is without conventional invasive ductal carcinoma cells (2). PMBC is represented by a mass with a $>90 \%$ mucinous component (3). MBC is linked with a more favorable prognosis, a longer disease-free interval and a lower incidence of axillary node metastasis compared with infiltrating ductal carcinoma-not otherwise specified (IDC-NOS) $(1,2,4)$. However, recurrence and metastasis of MBC are frequently present in clinical practice.

Angiogenesis is a prerequisite for tumor development; there is a close association between the formation of blood vessels in the vicinity of tumor cells and the potential for tumor formation, invasion and metastasis. Angiogenesis is induced and developed in response to two sets of extracellular signals: soluble angiogenic factors and the extracellular matrix (5). Breast carcinoma has been shown to be an angiogenesisdependent tumor through experimental and clinical data. Vascular endothelial growth factor (VEGF) is the most potent endothelial cell mitogen (6) and a regulator of vascular permeability, therefore, VEGF has been considered as a powerful novel prognostic tool (7). However, the associations between VEGF expression in MBC and IDC-NOS and the morphology, behavior and prognosis of tumors, and the differences between $\mathrm{MBC}$ and IDC-NOS, are unclear.

The good prognosis of MBC is closely associated with the formation of MUC around the cells (8). Previous studies have revealed the expression of the MUC1, MUC2, MUC3, MUC4, MUC5A and MUC6 proteins in PMBC, and this expression has been suggested to be a prognostic factor (9). Gel-forming secretory MUCs, including MUC2 and MUC6, exhibit a high expression rate in mucinous carcinoma, indicating that high production of these types of MUCs may act as a barrier to the extension of cancer, resulting in less aggressive biological behavior. However, the expression of 
MUC1, which is associated with a poor prognosis in gastric and colorectal cancer types, is low in MBC (9). A study by Ahmed (10) highlighted that the MUC of MBC is derived from cell breakdown. We hypothesize that significant cell apoptosis may exist in the MBC tissues and produce a large amount of mucus. The most well-known biochemical hallmark of early- and late-stage apoptosis is cysteine protease activation. Arginine-glycine-aspartate synthetic peptides induce apoptosis by direct caspase-3 activation (11). Caspase-3 is also required for the DNA fragmentation and morphological changes associated with apoptosis (12). A high level of active caspase-3 in cells and tissues is an important biomarker for apoptosis induced by a wide variety of apoptotic signals (13). Thus, detection of caspase- 3 expression can reflect the apoptotic status of tumor cells, which may aid in explaining the differences in prognosis and survival between MBC and IDC-NOS.

In the present study, the expression of VEGF and caspase-3 in MBC and IDC-NOS was investigated using immunohistochemical staining, and the association between the expression levels of VEGF and caspase-3 and clinicopathological features were further investigated.

\section{Materials and methods}

Patients and tissues. A total of 54 patients with MBC and 60 randomly selected patients with IDC-NOS who underwent surgery at the First Affiliated Hospital of China Medical University (Shenyang, Liaoning, China) between May 2009 and June 2011 were included in the present study. MBC is a rare type of breast cancer, so the 54 patients with $\mathrm{MBC}$ included all the MBC in accordance with the set of conditions between May 2009 and June 2011 who underwent surgery at the First Affiliated Hospital of China Medical University. Cases in which complete clinicopathological information and formalin-fixed, paraffin-embedded breast tissues could not be obtained were excluded. All patients had not received radiotherapy or chemotherapy prior to surgery. The diagnosis of all cases was confirmed according to the criteria of the World Health Organization (14), as assessed by the Department of Pathology (First Affiliated Hospital). Archival formalin-fixed paraffin-embedded breast tissues were retrieved. Patients were followed up for a median period of 47 months (range, 28-77 months) subsequent to the initial cancer surgery. Follow-up consisted of regular clinic visits and ultrasound of the breast and axillary node, supraclavicular area and infraclavicular region, with or without mammography, lung computed tomography, liver ultrasound, bone emission computed tomography and blood tests at the discretion of the treating specialist. Relevant clinical and pathological information are described in Table I.

Immunohistochemical staining. Immunohistochemical examination was performed on $4-\mu \mathrm{m}$ thick, formalin-fixed, paraffin-embedded sections using UltraSensitive ${ }^{\mathrm{TM}}$ SP IHC kit (MXB Co., Ltd., Fuzhou, China). Briefly, following deparaffinization (with xylene) and rehydration (with alcohol), the endogenous peroxidase activity was blocked with $3 \% \mathrm{H}_{2} \mathrm{O}_{2}$. Antigen retrieval was performed with a high-pressure cooker and normal serum (part of the UltraSensitive ${ }^{\mathrm{TM}}$ SP IHC kit) was applied to the sections at room temperature for $30 \mathrm{~min}$ to block non-specific antibody binding. The sections were then incubated overnight at $4^{\circ} \mathrm{C}$ with the primary antibodies, including monoclonal mouse-anti-human caspase-3 (1:50 dilution; catalog no. ab2171; Abcam, Cambridge, MA, USA) and monoclonal mouse-anti-human VEGF (1:300 dilution; catalog no. sc-7269; Santa Cruz Biotechnology, Inc., Dallas, TX, USA). Sections were further incubated with the biotin-labeled IgG secondary antibody solution from the UltraSensitive ${ }^{\mathrm{TM}}$ SP IHC kit at room temperature for $15 \mathrm{~min}$, followed by streptavidin-peroxidase incubation at room temperature for 15 min. Finally, sections were stained with 3,3-diaminobenzidine, counterstained with hematoxylin for $5 \mathrm{~min}$ and mounted. Negative controls were processed with PBS instead of the primary antibody.

Immunohistochemical scoring. The immunostained sections were assessed with an optical microscope at x400 magnification, based on manual counting of positive cells in each tissue by two observers blinded to clinical outcomes. Cases of disagreement were reviewed jointly to obtain a consensus score. The percentage of positive cells and staining intensity of VEGF and caspase-3 were scored. Intensity was graded as negative (score 0 ), weak (score 1), moderate (score 2) or strong (score 3), and percentage of positive cells was graded as $<5 \%$ (score 0), 5-25\% (score 1), 26-50\% (score 2), 51-75\% (score 3) and $>75 \%$ (score 4 ). The final score of VEGF and caspase-3 expression was determined by multiplying the intensity score and percentage score, with a range of 0-12. According to the scoring results, all patients were divided to two groups: Low (score of 0-5) and high (score of 6-12) expression.

Statistical analysis. Statistical analysis was performed using SPSS version 19.0 statistical software (IBM Corp., Armonk, New York, USA). A $\chi^{2}$ test and Fisher's exact test were used to identify the differences between MBC and IDC-NOS with regard to clinicopathological features, and for the associations between VEGF or caspase-3 and clinicopathological variables. Disease-free survival (DFS) was recorded from the date of surgery to the relapse date or the last follow-up date, and was estimated using the Kaplan-Meier analysis. The statistical significance of differential survival was assessed using the log-rank test. Cox regression analysis for DFS was used. $\mathrm{P}<0.05$ was used to indicate a statistically significant difference. Variables with a univariate $\mathrm{p}$-value of $<0.1$ were included in the multivariate model.

\section{Results}

Characteristics of the study population. A total of 54 female patients with MBC (median age 53.87 years; range, 24-82 years) and 60 IDC-NOS (median age 50.08 years; range, 27-78 years) were included. The cohort consisted of 94.5\% pathological tumor stage $1-2\left(\mathrm{pT}_{1-2}\right)$ patients, $5.6 \%$ $\mathrm{pT}_{3-4}$ patients and $16.8 \%$ node-positive patients in the MBC group, and $98.4 \% \mathrm{pT}_{1-2}$ patients, $1.7 \% \mathrm{pT}_{3-4}$ patients and $45.0 \%$ node-positive patients in the IDC-NOS group. No metastasis was observed in either group prior to surgery. In the MBC group, $92.6 \%$ of patients chose to undergo a mastectomy and $7.4 \%$ of patients chose breast-conserving 
Table I. Clinical and pathological features of the patients $(n=114)$.

\begin{tabular}{lccc}
\hline & MBC & IDC & \\
Characteristics & $(n=54)$ & $(n=60)$ & P-value \\
\hline
\end{tabular}

Age, years

Mean

$\leq 50, \mathrm{n}(\%)$

$>50, \mathrm{n}(\%)$

T stage, n $(\%)$

pT1

$\mathrm{pT} 2$

pT3-4

$\mathrm{N}$ stage, n (\%)

N0

N1

N2

N3

TNM stage, $\mathrm{n}(\%)$

I

II

III

ER status, n (\%)

Negative
Positive
PR status, n $(\%)$
Negative
Positive

Hormone receptor status, n (\%)

Negative
Positive

HER-2 status, n (\%)

Negative

Positive

Unknown

Ki-67, n (\%)

$\leq 20 \%$

$>20 \%$

p53, n (\%)

Negative

Positive

Unknown

Surgery

Mastectomy

BCS

Axillary operation

Sentinel lymph

node biopsy

Axillary clearance

Chemotherapy

No

Yes

$\begin{array}{cc}53.87 & 50.08 \\ 26(48.15) & 35(58.33) \\ 28(51.85) & 25(41.67) \\ & \\ 23(42.59) & 19(31.67) \\ 28(51.85) & 40(66.67) \\ 3(5.56) & 1(1.67)\end{array}$

0.079

0.276

0.203

$45(83.33) \quad 33(55.00)$

$5(9.26) \quad 15(25.00)$

$3(5.56) \quad 7(11.67)$

$1(1.85) \quad 5(8.33)$

$23(42.59) \quad 13(21.67)$

$27(50.00) \quad 35(58.33)$

$4(7.41) \quad 12(20.00)$

$6(11.11) \quad 23(38.33)$

48 (88.89) $\quad 37(61.67)$

$18(33.33) \quad 28(46.67)$

$36(66.67) \quad 32(53.33)$

$5(9.26) \quad 22(36.67)$

$49(90.74) \quad 38(63.33)$

$1(1.85) \quad 35(58.33)$

$43(79.63) \quad 10(16.67)$

$10(18.52) \quad 15(25.00)$

$41(75.93) \quad 30(50.00)$

$13(24.07) \quad 30(50.00)$

$19(35.19) \quad 22(36.37)$

$28(51.85) \quad 32(53.33)$

7 (12.96) $6(10.00)$

$50(92.59) \quad 58(96.67)$

$4(7.41) \quad 2(3.33)$

$8(14.81) \quad 2(3.33)$

$46(85.19) \quad 58(96.67)$

$12(22.22) \quad 2(3.33)$

$42(77.78) \quad 58(96.67)$

$0.010^{\mathrm{a}}$

$0.023^{\mathrm{a}}$

$0.001^{\mathrm{a}}$

0.147

$0.001^{\mathrm{a}}$

0.085

$0.004^{\mathrm{a}}$ $<0.001^{\mathrm{a}}$
Table I. Continued.

\begin{tabular}{lccc}
\hline Characteristics & $\begin{array}{c}\text { MBC } \\
(\mathrm{n}=54)\end{array}$ & $\begin{array}{c}\text { IDC } \\
(\mathrm{n}=60)\end{array}$ & P-value \\
\hline $\begin{array}{l}\text { Anthracycline } \\
\text { included }\end{array}$ & $31(57.41)$ & $13(21.67)$ & \\
$\begin{array}{l}\text { Taxane included } \\
\text { Anthracycline and } \\
\text { taxane included }\end{array}$ & $4(7.41)$ & $3(5.00)$ & \\
$\quad$ & $6(11.11)$ & $41(68.33)$ & \\
$\begin{array}{l}\text { Other } \\
\text { Radiotherapy }\end{array}$ & $1(1.85)$ & $1(1.67)$ & \\
No & $54(100.00)$ & $56(93.33)$ & \\
Yes & $0(0.00)$ & $4(6.67)$ &
\end{tabular}

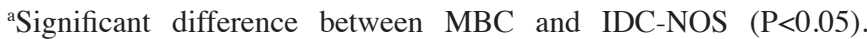
MBC, mucinous breast carcinoma; IDC-NOS, infiltrating ductal carcinoma-not otherwise specified; ER, estrogen receptor; PR, progesterone receptor; TNM, tumor-node-Metastasis; BCS, breast-conserving surgery; HER2, human epidermal growth factor receptor 2; p53, cellular tumor antigen p53.

Table II. VEGF and caspase-3 expression in MBC and IDC-NOS.

\begin{tabular}{lccc}
\hline Expression & $\begin{array}{c}\text { MBC, } \\
\mathrm{n}(\%)\end{array}$ & $\begin{array}{c}\text { IDC-NOS, } \\
\mathrm{n}(\%)\end{array}$ & P-value \\
\hline VEGF high & $23(42.59)$ & $37(61.67)$ & $0.042^{\mathrm{a}}$ \\
Caspase-3 high & $31(57.41)$ & $20(33.33)$ & $0.010^{\mathrm{a}}$
\end{tabular}

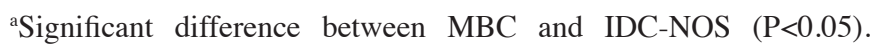
$\mathrm{MBC}$, mucinous breast carcinoma; IDC-NOS, infiltrating ductal carcinoma-not otherwise specified; VEGF, vascular endothelial growth factor.

surgery. Furthermore, $14.8 \%$ of patients underwent sentinel lymph node biopsy and $85.2 \%$ of patients received axillary clearance. In the MBC group, $22.2 \%$ of patients did not accept adjuvant chemotherapy and no patients accepted adjuvant radiotherapy following surgery. There were no significant differences in terms of age, $\mathrm{T}$ stage, breast surgery and adjuvant radiotherapy between the two groups. In contrast to IDC-NOS, the MBC patients showed a higher rate of positive ER and hormone receptor, and a larger population of which expression $\mathrm{Ki}-67$ was $\leq 20 \%$. MBC patients

0.420 tended to have significantly less lymph node metastasis and a lower tumor-node-metastasis (TNM) stage (15) compared with IDC-NOS patients $(\mathrm{P}=0.010$ and $\mathrm{P}=0.023$, respectively) (Table I).

VEGF and caspase-3 expression in MBC and IDC-NOS patients. The positive staining of VEGF and caspase-3 was mainly observed in the cytoplasm (Fig. 1). The expression of VEGF and caspase-3 was significantly different between the MBC and IDC-NOS patients. In total, $42.59 \%$ of MBC patients exhibited a high VEGF score $(\geq 6)$, with this 
A

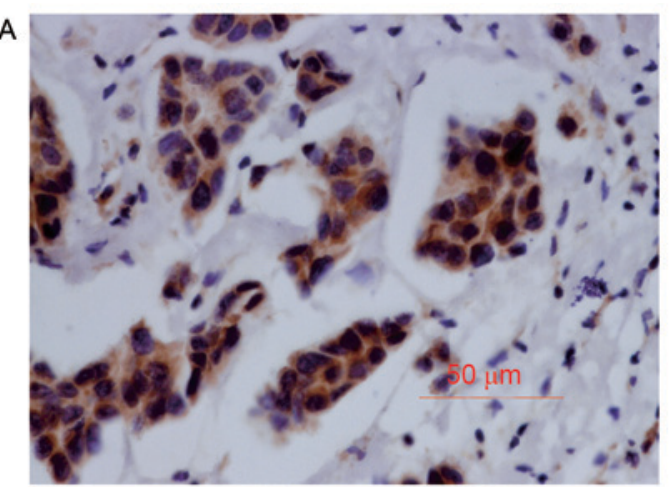

C

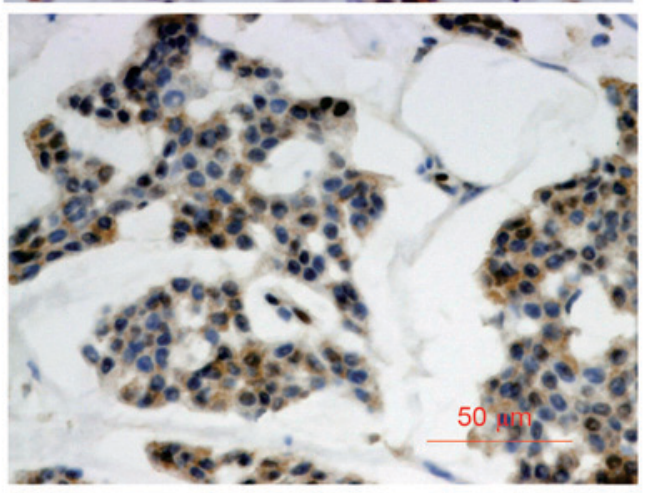

B

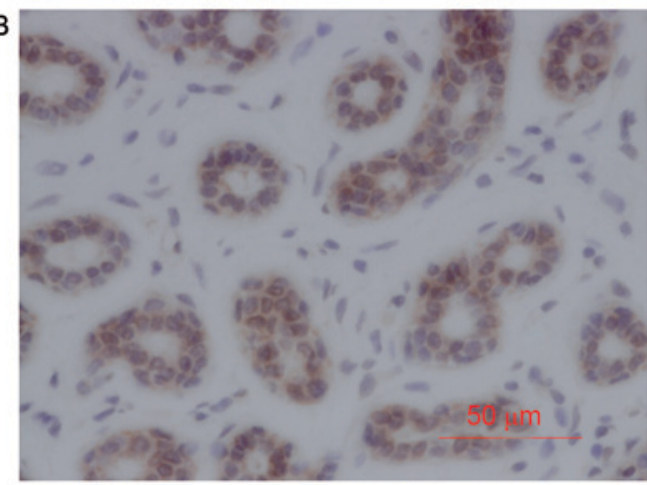

D

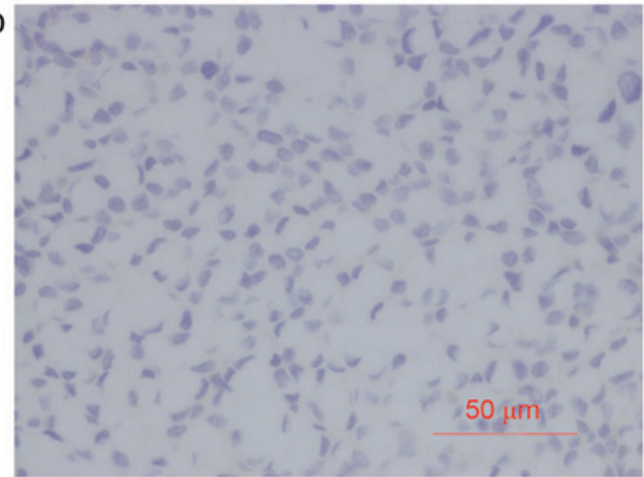

Figure 1. Expression of VEGF and caspase-3 in MBC and IDC-NOS. (A) The high expression of VEGF in MBC. (B) High expression of VEGF in IDC-NOS (C) The high expression of caspase-3 in MBC. (D) The low expression of caspase-3 in IDC-NOS. Scale bar, $50 \mu$ m; original magnification, $x 400$. VEGF, vascular endothelial growth factor; MBC, mucinous breast carcinoma.

A

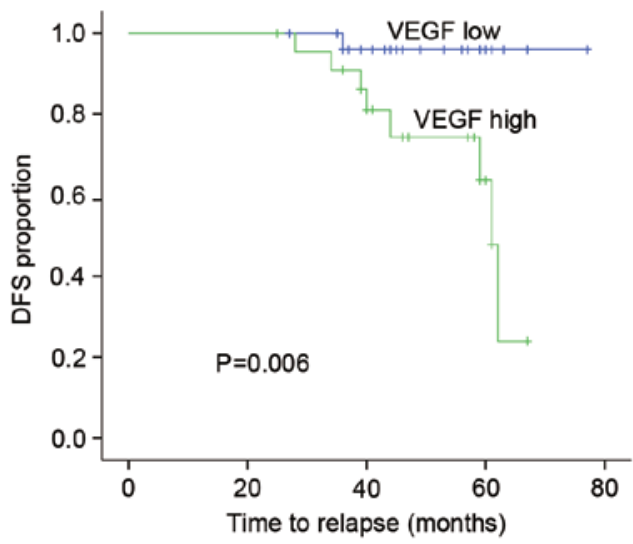

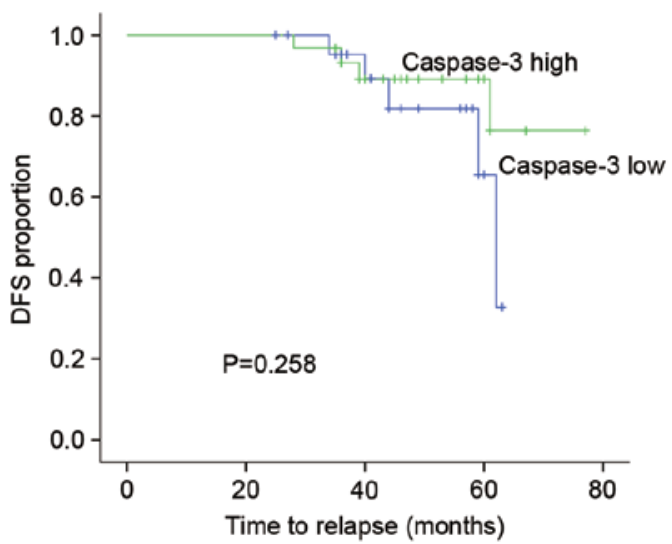

Figure 2. Kaplan-Meier survival analysis of DFS in MBC. (A) The high expression of VEGF was found to be significantly associated with a poor 5-year DFS rate in MBC $(\mathrm{P}<0.05)$. (B) The expression of caspase-3 exhibited no significant association with DFS in MBC. DFS, disease-free survival; VEGF, vascular endothelial growth factor; $\mathrm{MBC}$, mucinous breast carcinoma.

percentage being $61.67 \%$ in the IDC-NOS group $(\mathrm{P}=0.042)$. Furthermore, 31 cases $(57.4 \%)$ of MBC patients exhibited high caspase- 3 expression ( $\geq 6$ ), but only 20 cases (33.33\%) in the IDC-NOS group exhibited high caspase-3 expression $(\mathrm{P}=0.028)$ (Table II).

Association between VEGF and caspase-3 expression and $D F S$ in MBC patients. Since the expression, function and mechanism of IDC-NOS is already clear, the present study shows the associations between them in MBC. Kaplan-Meier log-rank test showed that the patients with high VEGF expression tended to experience shorter DFS times $(\mathrm{P}=0.006)$ compared with those with low expression in MBC. However, there was no association between caspase- 3 expression and DFS time in MBC patients (Fig. 2).

Association between VEGF and caspase-3 expression and clinicopathological variables in MBC patients. There was a significant association between VEGF expression and age, nodal status and TNM stage in the MBC patients, but there was no significant association between caspase-3 expression and age, tumor stage, nodal status, TNM stage, estrogen 
Table III. Association between VEGF, caspase-3 and other variables in MBC.

\begin{tabular}{|c|c|c|c|c|c|c|}
\hline Variables & VEGF (-) & VEGF (+) & P-value & Caspase-3 (-) & Caspase-3 (+) & P-value \\
\hline Age, years & & & $0.013^{\mathrm{a}}$ & & & 0.783 \\
\hline$\leq 50$ & 20 & 7 & & 12 & 15 & \\
\hline$>50$ & 11 & 16 & & 11 & 16 & \\
\hline Tumor stage & & & 0.220 & & & 0.658 \\
\hline pT1 & 11 & 12 & & 9 & 14 & \\
\hline pT2-3 & 20 & 11 & & 14 & 17 & \\
\hline Nodal status & & & $0.002^{\mathrm{a}}$ & & & 0.717 \\
\hline Negative & 30 & 15 & & 20 & 25 & \\
\hline Positive & 1 & 8 & & 3 & 6 & \\
\hline TNM stage & & & $0.008^{\mathrm{a}}$ & & & 1.000 \\
\hline I-IIa & 30 & 16 & & 20 & 26 & \\
\hline IIb-IIIIc & 1 & 7 & & 3 & 5 & \\
\hline ER & & & 1.000 & & & 0.384 \\
\hline Negative & 3 & 3 & & 4 & 2 & \\
\hline Positive & 28 & 20 & & 19 & 29 & \\
\hline PR & & & 0.107 & & & 0.554 \\
\hline Low $^{\mathrm{b}}$ & 12 & 14 & & 10 & 16 & \\
\hline $\mathrm{High}^{\mathrm{c}}$ & 19 & 9 & & 13 & 15 & \\
\hline Ki-67, \% & & & 0.358 & & & 0.346 \\
\hline$\leq 20$ & 22 & 19 & & 16 & 25 & \\
\hline$>20$ & 9 & 4 & & 7 & 6 & \\
\hline
\end{tabular}

${ }^{a}$ Significant difference between MBC and IDC-NOS (P<0.05); ${ }^{\text {p }} \mathrm{PR}<20 \%$; ${ }^{\mathrm{c}} \mathrm{PR} \geq 20 \%$. MBC, mucinous breast carcinoma; VEGF, vascular endothelial growth factor; ER, estrogen receptor; PR, progesterone receptor.

receptor (ER) status, progesterone receptor $(\mathrm{PR})$ status or Ki-67 expression (Table III).

Cox analysis in $M B C$. Univariate Cox regression analyses showed higher VEGF score, positive nodal status and higher TNM stage were significant predictors of worse DFS. Caspase-3 expression had no significant predictive value in terms of DFS. Moreover, multivariate analysis analyzed the correlation between DFS and VEGF expression, nodal status and TNM stage, and found that TNM stage was significantly associated with worse DFS (Table IV). Ki-67 expression was also analyzed. The rate of $\mathrm{Ki}-67(\leq 20 \%)$ expression was $75.93 \%$ in the $\mathrm{MBC}$ patients compared with $50 \%$ in the IDC-NOS patients $(\mathrm{P}<0.05)$, suggesting that MBC tumors may have lower proliferative ability than IDC-NOS tumors. However, Cox survival analysis showed that Ki-67 expression was not significantly associated with DFS in the MBC patients.

\section{Discussion}

The majority of previous MBC clinical studies found that MBC showed higher ER- and PR-positive rates, less lymph node metastasis, lower TNM stage and notably higher OS and DFS rates (16-20). The present study analyzed the basic information of MBC patients and compared it with that from IDC-NOS patients treated in the same period. The results were consistent with those of the previous studies, in which the patients with MBCs had a better prognosis than those with IDC-NOS.

Jao et al (21) studied 7 cases of MBC using electron microscopy and found that in addition to the abundant production of mucosubstance, MBC also featured the absence of myoepithelial differentiation and basal lamina deposition, the presence of notably developed cytoplasmic filamentous systems, a relatively scarcity of lysosomes, apparently and frequently well-developed intercellular junctions and a marked paucity of stromal vessels. These data suggest that the favorable clinical prognosis of MBC may be the result of multiple complicated factors (21).

Tumor growth requires constant vascular growth and remodeling so that solid tumors can exceed $1-2 \mathrm{~mm}^{3}$ in size. VEGF and its receptors are key regulators of angiogenesis, meaning that they are attractive therapeutic targets (22). Microvessel density and tumor VEGF expression in hepatocellular carcinoma have previously been assessed, and the results indicated that upregulation of VEGF promoted angiogenesis, tumor growth and intrahepatic metastasis (23). VEGF-C-producing cancer cells may induce lymphatic vessel proliferation and dilation, resulting in cancer cell invasion into the lymphatic vessels and lymph node metastasis (24). Cancer treatment using a number of VEGF-targeted inhibitory agents is currently being assessed. VEGF-targeted therapy has been approved for the clinical treatment of metastatic triple-negative breast cancer (25). The paucity of stromal vessels in MBC may 
Table IV. Cox univariate analysis and multivariate analysis of clinicopathological variables, including VEGF, for DFS in MBC.

\begin{tabular}{|c|c|c|c|c|c|c|}
\hline \multirow[b]{2}{*}{ Variables } & \multicolumn{3}{|c|}{ Univariate analysis } & \multicolumn{3}{|c|}{ Multivariate analysis } \\
\hline & HR & $95 \% \mathrm{CI}$ & P-value & HR & $95 \% \mathrm{CI}$ & P-value \\
\hline \multicolumn{7}{|l|}{ VEGF } \\
\hline High & 10.640 & $(1.326-85.403)$ & $0.026^{\mathrm{a}}$ & 5.881 & $(0.632-54.741)$ & 0.120 \\
\hline Low & & & & & & \\
\hline \multicolumn{7}{|l|}{ Caspase-3 } \\
\hline High & 0.473 & $(0.125-1.785)$ & 0.269 & & & \\
\hline Low & & & & & & \\
\hline \multicolumn{7}{|l|}{ Age, years } \\
\hline$\leq 50$ & 1.809 & $(0.452-7.244)$ & 0.402 & & & \\
\hline$>50$ & & & & & & \\
\hline \multicolumn{7}{|c|}{ Tumor stage } \\
\hline $\mathrm{pT} 2 / \mathrm{pT} 3$ & 1.941 & $(0.479-7.863)$ & 0.353 & & & \\
\hline pT1 & & & & & & \\
\hline \multicolumn{7}{|c|}{ Nodal status } \\
\hline Positive & 5.844 & $(1.452-23.529)$ & $0.013^{\mathrm{a}}$ & 0.477 & $(0.060-3.809)$ & 0.485 \\
\hline Negative & & & & & & \\
\hline \multicolumn{7}{|l|}{ TNM } \\
\hline II/III & 11.689 & $(2.762-49.465)$ & $0.001^{\mathrm{a}}$ & 10.386 & $(1.230-87.703)$ & $0.032^{\mathrm{a}}$ \\
\hline $\mathrm{I}$ & & & & & & \\
\hline \multicolumn{7}{|l|}{ ER } \\
\hline Positive & 0.872 & $(0.172-4.409)$ & 0.868 & & & \\
\hline Negative & & & & & & \\
\hline \multicolumn{7}{|l|}{ PR } \\
\hline High $^{\mathrm{b}}$ & 0.542 & $(0.135-2.618)$ & 0.386 & & & \\
\hline \multicolumn{7}{|l|}{ Low $^{\mathrm{c}}$} \\
\hline \multicolumn{7}{|l|}{$\mathrm{Ki}-67, \%$} \\
\hline$>20$ & 1.299 & $(0.262-6.454)$ & 0.749 & & & \\
\hline$\leq 20$ & & & & & & \\
\hline
\end{tabular}

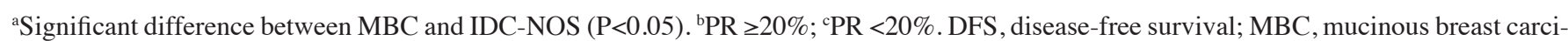
noma; VEGF, vascular endothelial growth factor; ER, estrogen receptor; PR, progesterone receptor; HR, hazard ratio; CI, confidence interval.

be an important adverse factor for angiogenesis (21). In the present study, VEGF expression was assessed in MBC and IDC-NOS samples, and VEGF expression in MBC was found to be lower than that in IDC-NOS. It was concluded that low VEGF expression in MBC may be associated with the paucity of tumor vessels and result in a better prognosis. In the MBC patients, high VEGF expression was significantly associated with primary lymph node metastasis and high TNM stage. Kaplan-Meier curves showed that VEGF was associated with DFS in the MBC patients, while in the Cox multivariate analysis model, only TNM stage was the independent prognostic factor.

A large amount of mucus is a typical feature of MBC samples. Norris and Taylor (8) found that the mucus in the tumor tissues plays an important role in the prognosis of the patients and the whole clinical course of the disease. Ahmed (10) noted that the formation of mucus is caused by the breakdown of cancer cells, followed by the degeneration of mitochondria, and that it leads to a marked decrease in tumor invasion. In addition to necrosis, apoptosis is a large part of cell disintegration. The caspases are a family of genes that are important for the maintenance of homeostasis via the regulation of cell death and inflammation (26). Caspase-3 is the key molecular factor in various apoptotic pathways $(27,28)$. The present study examined caspase-3 expression in each group. MBC samples showed high caspase-3 expression, suggesting that caspase-3-mediated apoptosis may hinder tumor progression in MBC patients. Unexpectedly, caspase-3 expression was not associated with DFS or other clinicopathological parameters in the MBC patients.

The Ki-67 index has potential prognostic and predictive value in breast cancer, and has become an important, routinely used proliferation biomarker (29). The present study analyzed the expression of $\mathrm{Ki}-67$ and found that the rate of $\mathrm{Ki}-67$ ( $\leq 20 \%$ ) expression in MBC was $75.93 \%$ compared with $50 \%$ in IDC-NOS. A significant difference exists between MBC and 
IDC-NOS, which suggests that tumor cells of MBC may have a lower proliferation ability than those of IDC-NOS. However, Cox survival analysis showed that Ki-67 expression was not directly associated with DFS in MBC patients.

In conclusion, the present study revealed that high rate of hormone receptor and caspase-3, low expression of VEGF and Ki-67 and earlier TNM stage may contribute to improved prognosis of MBC compared with IDC-NOS. VEGF and caspase-3 may serve a role in mucus production, which is important in MBC progression. However, neither high expression of VEGF nor caspase-3 had a significant direct association with the DFS of patients with MBC. The mucinous breast cancer cell lines may need to be cultured in the future to explore the proliferation, invasion and migration ability of cancer cells and the exact role of mucus in tumor progression.

\section{Acknowledgements}

The present study was supported by grants from the National Natural Science Foundation of China (grant no. 81172199).

\section{References}

1. Dumitru A, Procop A, Iliesiu A, Tampa M, Mitrache L, Costache M, Sajin M, Lazaroiu A and Cirstoiu M: Mucinous breast cancer: A review study of 5 year experience from a hospital-based series of cases. Maedica (Buchar) 10: 14-18, 2015.

2. Ranade A, Batra R, Sandhu G, Chitale RA and Balderacchi J: Clinicopathological evaluation of 100 cases of mucinous carcinoma of breast with emphasis on axillary staging and special reference to a micropapillary pattern. J Clin Pathol 63 1043-1047, 2010.

3. Komaki K, Sakamoto G, Sugano H, Morimoto T and Monden Y: Mucinous carcinoma of the breast in Japan. A prognostic analysis based on morphologic features. Cancer 61: 989-996, 1988.

4. Ha KY, Deleon P and Deleon W: Invasive mucinous carcinoma of the breast. Proc (Bayl Univ Med Cent) 26: 295-297, 2013.

5. Le Querrec A, Duval D and Tobelem G: Tumour angiogenesis. Baillieres Clin Haematol 6: 711-730, 1993.

6. Yancopoulos GD, Davis S, Gale NW, Rudge JS, Wiegand SJ and Holash J: Vascular-specific growth factors and blood vessel formation. Nature 407: 242-248, 2000.

7. Gasparini G: Prognostic value of vascular endothelial growth factor in breast cancer. The oncologist 5 Suppl 1: 37-44, 2000.

8. Norris HJ and Taylor HB: Prognosis of mucinous (Gelatinous) carcinoma of the breast. Cancer 18: 879-885, 1965.

9. Matsukita S, Nomoto M, Kitajima S, Tanaka S, Goto M, Irimura T, Kim YS, Sato E and Yonezawa S: Expression of mucins (MUC1, MUC2, MUC5AC and MUC6) in mucinous carcinoma of the breast: Comparison with invasive ductal carcinoma. Histopathology 42: 26-36, 2003.

10. Ahmed A: The myoepithelium in human breast carcinoma. J Pathol 113: 129-135, 1974

11. Buckley CD, Pilling D, Henriquez NV, Parsonage G, Threlfall K, Scheel-Toellner D, Simmons DL, Akbar AN, Lord JM and Salmon M: RGD peptides induce apoptosis by direct caspase-3 activation. Nature 397: 534-539, 1999.
12. Jänicke RU, Sprengart ML, Wati MR and Porter AG: Caspase-3 is required for DNA fragmentation and morphological changes associated with apoptosis. J Biol Chem 273: 9357-9360, 1998.

13. Mazumder S, Plesca D and Almasan A: Caspase-3 activation is a critical determinant of genotoxic stress-induced apoptosis. Methods Mol Biol 414: 13-21, 2008.

14. Frank GA, Danilova NV, Andreeva IuIu and Nefedova NA: WHO classification of tumors of the breast, 2012. Arkh Patol 75: 53-63, 2013 (In Russian).

15. Edge SB, Byrd DR and Compton CC (eds): AJCC Cancer Staging Manual. 7th edition. Springer, New York, NY, 2010.

16. Kashiwagi S, Onoda N, Asano Y, Noda S, Kawajiri H, Takashima T, Ohsawa M, Kitagawa S and Hirakawa K: Clinical significance of the sub-classification of 71 cases mucinous breast carcinoma. Springerplus 2: 481, 2013.

17. Zekioglu O,Erhan Y,Ciris M and Bayramoglu H: Neuroendocrine differentiated carcinomas of the breast: A distinct entity. Breast 12: 251-257, 2003.

18. Bae SY, Choi MY, Cho DH, Lee JE, Nam SJ and Yang JH: Mucinous carcinoma of the breast in comparison with invasive ductal carcinoma: Clinicopathologic characteristics and prognosis. J Breast Cancer 14: 308-313, 2011.

19. Park S, Koo J, Kim JH, Yang WI, Park BW and Lee KS: Clinicopathological characteristics of mucinous carcinoma of the breast in Korea: Comparison with invasive ductal carcinoma-not otherwise specified. J Korean Med Sci 25: 361-368, 2010.

20. Tseng HS, Lin C, Chan SE, Chien SY, Kuo SJ, Chen ST, Chang TW and Chen DR: Pure mucinous carcinoma of the breast: Clinicopathologic characteristics and long-term outcome among Taiwanese women. World J Surg Oncol 11: 139, 2013.

21. Jao W, Lao IO, Chowdhury LN and Gould VE: Ultrastructural aspects of mucinous (colloid) breast carcinoma. Diagn Gynecol Obstet 2: 83-92, 1980.

22. Rosen LS: VEGF-targeted therapy: Therapeutic potential and recent advances. Oncologist 10: 382-391, 2005.

23. Ng IO, Poon RT, Lee JM, Fan ST, Ng M and Tso WK: Microvessel density, vascular endothelial growth factor and its receptors Flt-1 and Flk-1/KDR in hepatocellular carcinoma. Am J Clin Pathol 116: 838-845, 2001.

24. Yonemura Y, Endo Y, Fujita H, Fushida S, Ninomiya I, Bandou E, Taniguchi K, Miwa K, Ohoyama S, Sugiyama K and Sasaki T: Role of vascular endothelial growth factor $\mathrm{C}$ expression in the development of lymph node metastasis in gastric cancer. Clin Cancer Res 5: 1823-1829, 1999.

25. Hein A, Lambrechts D, von Minckwitz G, Häberle L, Eidtmann H, Tesch H, Untch M, Hilfrich J, Schem C, Rezai M et al: Genetic variants in VEGF pathway genes in neoadjuvant breast cancer patients receiving bevacizumab: Results from the randomized phase III GeparQuinto study. Int J Cancer 137: 2981-2988, 2015.

26. McIlwain DR, Berger T and Mak TW: Caspase functions in cell death and disease. Cold Spring Harb Perspect Biol 5: a008656, 2013.

27. Fuchs $Y$ and Steller H: Programmed cell death in animal development and disease. Cell 147: 742-758, 2011.

28. Huang Q, Li F, Liu X, Li W, Shi W, Liu FF, O'Sullivan B, He Z, Peng Y, Tan AC, et al: Caspase 3-mediated stimulation of tumor cell repopulation during cancer radiotherapy. Nat Med 17: 860-866, 2011

29. Kontzoglou K, Palla V, Karaolanis G, Karaiskos I, Alexiou I, Pateras I, Konstantoudakis K and Stamatakos M: Correlation between Ki67 and breast cancer prognosis. Oncology 84: 219-225, 2013. 